\title{
Soft-QCD and diffractive processes at CMS and TOTEM
}

\author{
Deniz Sunar $\operatorname{Cerci}^{a, b, *}$ \\ a Adiyaman University, \\ Faculty of Arts and Sciences, Department of Physics, 02040 Adiyaman, Turkey \\ ${ }^{b}$ On behalf of the CMS and TOTEM Collaborations \\ E-mail: deniz.sunar .cerci@cern.ch
}

\begin{abstract}
At collider experiments, forward detectors with their kinematical acceptance allow for dedicated physics measurements. The recent measurements on soft QCD and diffractive processes performed with the CMS and TOTEM experiments are presented. The results are compared to theoretical predictions implemented in various Monte Carlo simulations as well as other measurements. These studies are useful to test predictions based on perturbative and non-perturbative QCD techniques and provide valuable inputs for tuning of Monte Carlo event generators.
\end{abstract}

The Eighth Annual Conference on Large Hadron Collider Physics-LHCP2020

25-30 May, 2020

online

${ }^{*}$ Speaker 


\section{Introduction}

In this paper, the recent CMS [1] and TOTEM [2] measurements on soft QCD and diffraction physics as well as the comparisons to predictions of various theoretical models are presented.

\section{Double parton scattering using same-sign $W$ boson pairs}

Double parton scattering (DPS) processes where each proton has two active partons produce exactly two different hard sub-processes shed light on the spatial structure of the hadrons and on the multi-parton correlations in the hadronic wave function. A study of WW production from DPS processes is performed with the CMS experiment by using pp collisions at $\sqrt{s}=13 \mathrm{TeV}$ [3]. In order to discriminate the signal from the dominant background processes, multivariate classifiers are used. The obtained value of WW production cross section via DPS using same sign final states along with their combination is shown in Figure 1. The experimental determination of the DPS WW cross section is achieved for the first time and extracted as $1.41 \pm 0.28$ (stat.) \pm 0.28 (syst.) pb with an observed significance of 3.9 standard deviations. This cross section leads to an effective cross section parameter of $\sigma_{\text {eff }}=12.7_{-2.9}^{+5.0} \mathrm{mb}$. The results obtained in this measurement constitute the first evidence for WW production from DPS.

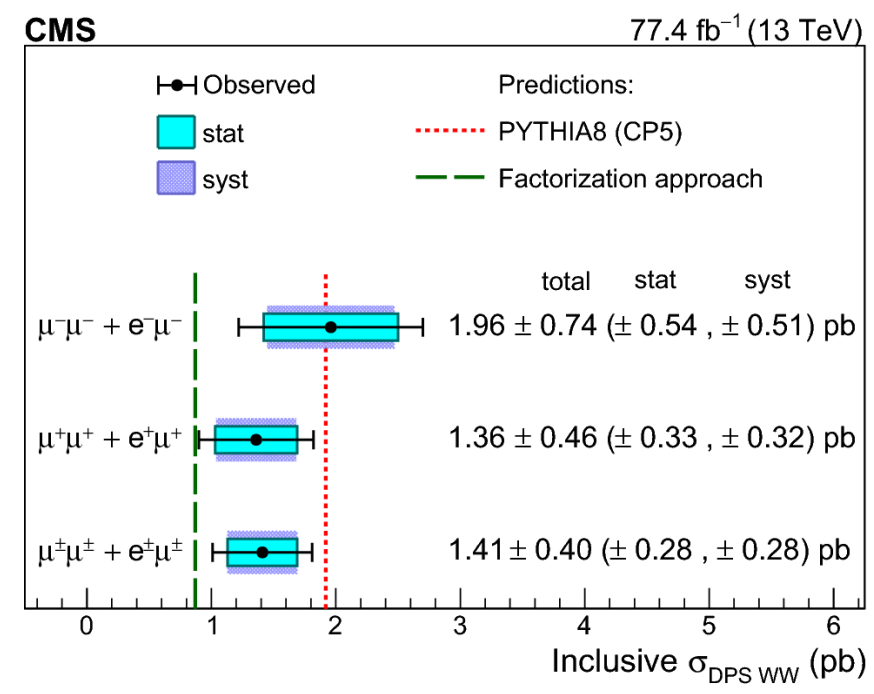

Figure 1: The observed DPS cross sections from the same-sign WW production analysis at $13 \mathrm{TeV}$ of CMS [3]. The statistical and systematical uncertainties ar e presented with shaded bands.

\section{Average very forward energy as a function of the track multiplicity}

The first correlation study of hadron activity at very forward and central rapidities is performed by the CMS Collaboration at $\sqrt{s}=13 \mathrm{TeV}$ [4]. The total energy deposited in the very forward region $(-6.6<\eta<-5.2)$ covered by the CASTOR calorimeter is obtained by summing the energy measured in each calorimeter tower above the noise threshold. The measurement is presented in terms of the total energy as well as its electromagnetic and hadronic components as a function 
of the central track multiplicity. The data are compared to various model predictions including different parameter tunes as well as entirely different physics approaches. The average total energy reconstructed in the CASTOR region increases with the track multiplictiy. This demonstrates that the underlying event tu ne parameters determined at central rapidities can be extrapolated to the very forward rapidity region within experimental uncertainties.

\section{Single-diffractive dijet production with proton tagging}

In pp collisions, diffractive processes give a significant contribution to the total cross section. Such events are characterised by a large rapidity gap around the protons, out of which at least one of the two stays intact or excited into a low-mass state, with only a small energy loss. When a hard scale process is present, diffraction can be described in terms of convolution of diffractive parton distribution functions and hard scattering cross sections calculated from perturbative quantum chromodynamics. However, soft rescattering effects occurring between the spectator partons break the factorisation. This leads to the suppression of diffractive cross sections where the suppression factor is called rapidity gap survival probability $\left\langle S^{2}\right\rangle$. The single-diffractive dijet production cross section in pp collisions at $\sqrt{s}=8 \mathrm{TeV}$ is measured as a function of the proton fractional momentum loss $\xi$ and the squared four momentum transfer at the proton vertex $t$ [5]. Events with at least two jets, each with transverse momentum $p_{T}>40 \mathrm{GeV}$ and pseudorapidity $|\eta|<4.4$ recorded by the CMS detector and the scattered proton found in the TOTEM roman pots are considered. The differential cross section of the single diffractive di-jet production as a function of $t$ and $\xi$ is shown in Figure 2 . The measured cross sections are compared to various predictions and found to be in agreement with the POMWIG model where the rapidity gap survival probabilities $\left\langle S^{2}\right\rangle$ of 1 and $7.4 \%$ are applied. The integrated cross section is measured as $\sigma_{j j}^{p X}=21.7 \pm 0.9$ (stat) ${ }_{-3.3}^{+3.0}$ athrm (syst) \pm 0.9 (lumi) nb.
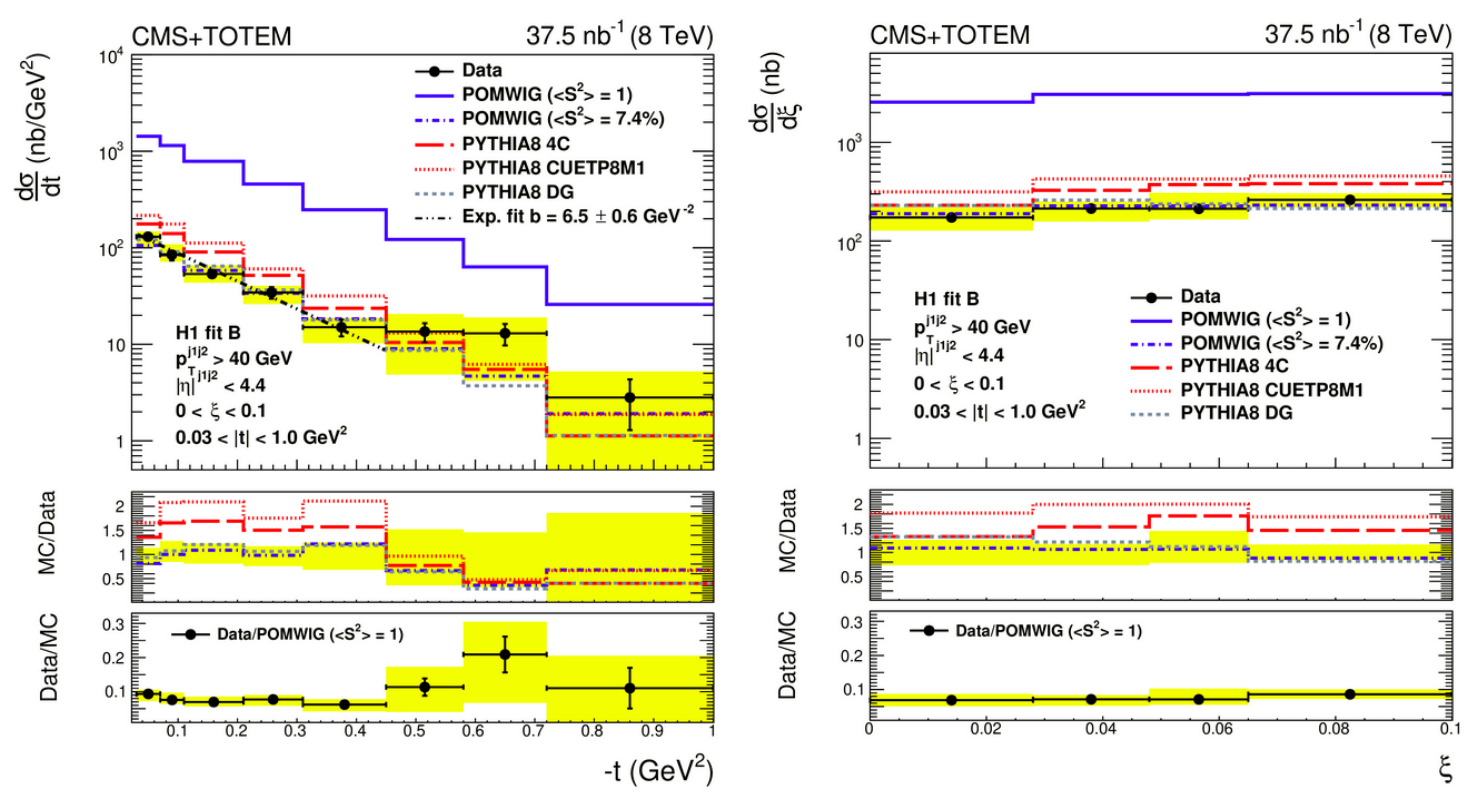

Figure 2: Differential cross section of the single diffractive di-jet production as a function of $t$ (left) and $\xi$ (right), together with the corresponding Monte Carlo predictions [5]. 


\section{Hard color singlet exchange in dijet events}

One of the processes sensitive to Balitsky-Fadin-Kuraev-Lipatov (BFKL) dynamics is the production of two jets separated by a large rapidity interval devoid of particle activity, known as Mueller-Tang jets [6] or jet-gap-jet events. The CMS detector with its unprecedented center-of-mass energy and large detector coverage in rapidity provides an ideal tool for testing BFKL dynamics and understanding the role of diffraction at large momentum transfers in strong nuclear interactions. The study [7] is performed with the low instantaneous luminosity data collected in pp collisions at $\sqrt{s}=13 \mathrm{TeV}$ by the CMS and TOTEM detectors. The fraction of jet-gap-jet events to events where the two jets have similar kinematics, $f_{C S E}$, is measured as a function of the pseudorapidity difference between the leading two jets, $\Delta \eta_{j j}$, and the transverse momentum of the subleading jet, $p_{T, j e t-2}$ (Figure 3). An increase with $\Delta \eta_{j j}$ and a weak dependency on $p_{T, j e t-2}$ are observed. The present analysis sets a constraint on the theoretical treatment of rapidity gap survival probability.
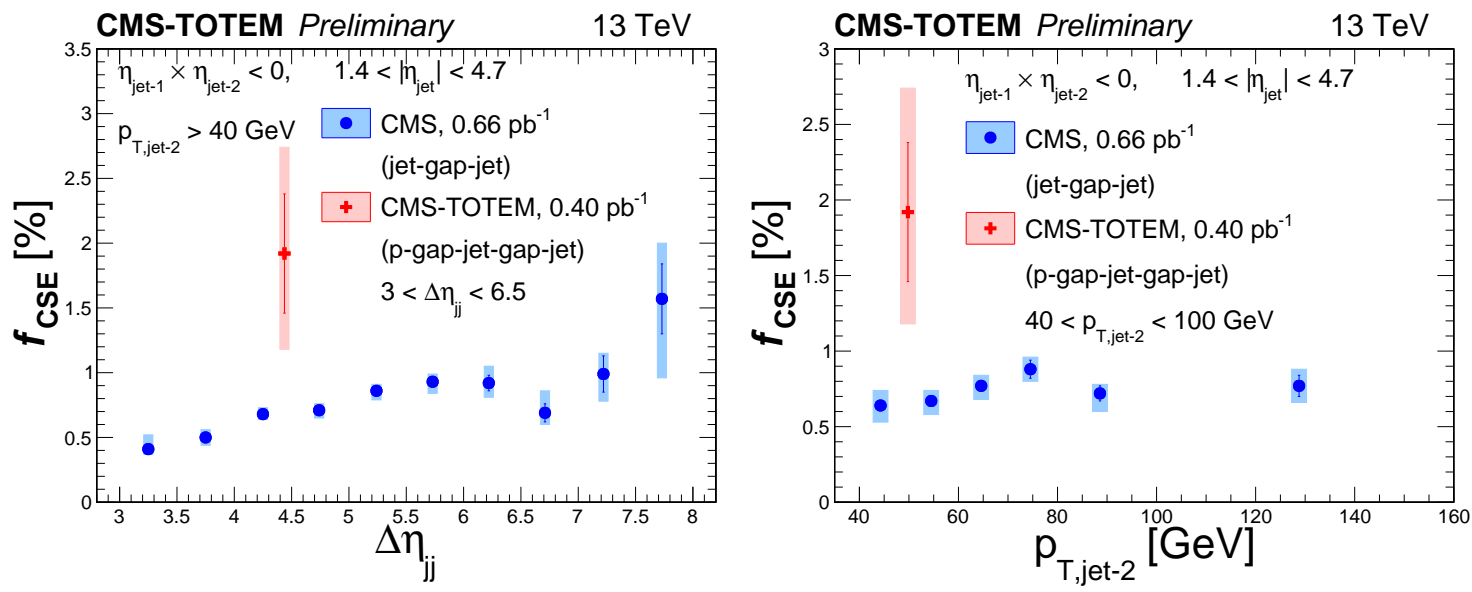

Figure 3: Gap fraction, $f_{C S E}$, measured as a function of $\Delta \eta_{j j}$ and $p_{T, j e t-2}$ in inclusive dijet events and in dijet events with a leading proton [7].

\section{References}

[1] CMS collaboration, The CMS experiment at the CERN LHC, JINST 3 (2008) S08004.

[2] TOTEM collaboration, The TOTEM experiment at the CERN Large Hadron Collider, JINST 3 (2008) S08007.

[3] CMS collaboration, Evidence for WW production from double-parton interactions in proton-proton collisions at $\sqrt{s}=13 \mathrm{TeV}$, Eur. Phys. J. C80 (2020).

[4] CMS collaboration, Measurement of the average very forward energy as a function of the track multiplicity at central pseudorapidities in proton-proton collisions at $\sqrt{s}=13 \mathrm{TeV}$, Eur. Phys. J. C79 (2019) 893 [arXiv: 1908.01750]. 
[5] CMS AND TOTEM collaboration, Measurement of single-diffractive dijet production in proton-proton collisions at $\sqrt{s}=8 \mathrm{TeV}$ with the CMS and TOTEM experiments, Submitted to Eur. Phys. J. C. (2020) [arXiv:2002.12146].

[6] A. Mueller and W.-K. Tang, High energy parton-parton elastic scattering in QCD, Physics Letters B 284 (1992) 123.

[7] CMS collaboration, Study of hard color singlet exchange in dijet events with proton-proton collisions at $\sqrt{s}=13 \mathrm{TeV}$, CMS-PAS-SMP-19-006 (2020) . 